\title{
A note on forced oscillations in differential equations with jumping nonlinearities
}

\author{
A. Buică · J. Llibre • O. Makarenkov
}

\begin{abstract}
The goal of this paper is to study bifurcations of asymptotically stable $2 \pi$-periodic solutions in the forced asymmetric oscillator $\ddot{u}+\varepsilon c \dot{u}+u+$ $\varepsilon a u^{+}=1+\varepsilon \lambda \cos t$ by means of a Lipschitz generalization of the second Bogolubov's theorem due to the authors. The small parameter $\varepsilon>0$ is introduced in such a way that any solution of the system corresponding to $\varepsilon=0$ is $2 \pi$-periodic. We show that exactly one of these solutions whose amplitude is $\frac{\lambda}{\sqrt{a^{2}+c^{2}}}$ generates a branch of $2 \pi$-periodic solutions when $\varepsilon>0$ increases. The solutions of this branch are asymptotically stable provided that $c>0$.
\end{abstract}

Keywords Asymptotic stability · Periodic solutions · Jumping nonlinearity · Method of averaging

\section{Introduction}

The differential equation for the coordinate $u$ of the mass attached via nonlinear spring to an immovable beam drawn at Fig. 1 reads as

$$
m \ddot{u}+c \dot{u}+k_{1} u+k_{2} u^{+}=f(t),
$$

where $f$ is a force applied to the mass in the vertical direction, see $[1,15,11]$.

The case where equation (1) takes the form

$$
m \ddot{u}+\varepsilon c_{\varepsilon} \dot{u}+k_{1} u+k_{2} u^{+}=\varepsilon f(t),
$$

A. Buică

Department of Mathematics, Babeş-Bolyai University, Cluj-Napoca, 400084, Romania

J. Llibre

Departament de Matemàtiques, Universitat Autònoma de Barcelona, Bellaterra, Barcelona, 08193, Catalonia, Spain

O. Makarenkov (corresponding author)

Department of Mathematical Sciences, University of Texas at Dallas, 800 West Campbell Road, Richardson, TX 75080, USA

Tel: +1 972-883-4617 Fax: +1 972-883-6622 


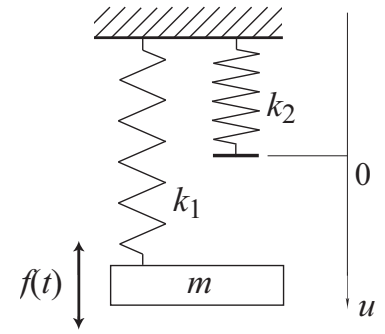

(a)

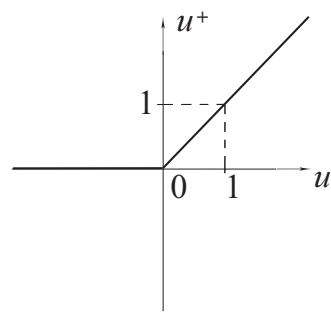

(b)

Fig. 1 (a) A driven mass attached to an immovable beam via a spring with piecewise linear stiffness, (b) the jumping nonlinearity $u \mapsto u^{+}$.

where $c_{\varepsilon} \rightarrow 0$ as $\varepsilon \rightarrow 0$, the bifurcation of asymptotically stable periodic solutions is studied in Glover-Lazer-McKenna [8]. In the setting where the unperturbed system $m \ddot{u}+k_{1} u+k_{2} u^{+}=0$ has a $T$-periodic orbit $u_{0}$, these authors related the existence of asymptotically stable $T$-periodic solutions near $u_{0}([0, T])$ to the existence of $\alpha \in[0, T]$ such that $\int_{0}^{T} \dot{u}_{0}(\tau) f(\tau-\alpha) d \tau=0$ and $d=\int_{0}^{T} \ddot{u}_{0}(\tau) f(\tau-\alpha) d \tau>0$. By the other words, the authors of [8] showed that the conclusion of the second Bogolubov's theorem holds for equation (2), even though it is not $C^{1}$. We quote this theorem for completeness, see [4].

Second Bogolubov's theorem. Consider the perturbed system

$$
\dot{x}=\varepsilon g(t, x, \varepsilon),
$$

where $g \in C^{1}\left(\mathbb{R} \times \mathbb{R}^{n} \times[0,1], \mathbb{R}^{n}\right)$ is T-periodic in the first variable. If $v_{0} \in \mathbb{R}^{n}$ is a zero of the bifurcation function

$$
g_{0}(v)=\int_{0}^{T} g(\tau, v, 0) d \tau
$$

and $\operatorname{det}\left(g_{0}\right)^{\prime}\left(v_{0}\right) \neq 0$, then for any $\varepsilon>0$ sufficiently small system (3) has a unique T-periodic solution $x_{\varepsilon}$ such that $x_{\varepsilon}(0) \rightarrow v_{0}$ as $\varepsilon \rightarrow 0$. If, in addition, all the eigenvalues of the matrix $\left(g_{0}\right)^{\prime}\left(v_{0}\right)$ have negative real part, then $x_{\varepsilon}$ is asymptotically stable.

Note, the change of variables

$$
\left(\begin{array}{l}
u(t) \\
\dot{u}(t)
\end{array}\right)=\left(\begin{array}{ll}
\cos t & \sin t \\
-\sin t & \cos t
\end{array}\right)\left(\begin{array}{l}
x_{1}(t) \\
x_{2}(t)
\end{array}\right)
$$

transforms equation (2) to the standard form (3) of averaging theory (see a similar example in Section 3).

In the next section of the paper we discuss a general class of Lipschitz systems (which includes, in particular, equation (2)) for which the conclusion of the second Bogolubov's theorem holds. The Lipschitz analogue of the second Bogolyubov's theorem (theorem 1) is then applied to the asymmetric oscillator

$$
\ddot{u}+\varepsilon c \dot{u}+u+\varepsilon a u^{+}=\varepsilon \lambda \cos t
$$


in section 3, where we obtain (theorem 2) explicit conditions for the coefficients $c, a$ and $\lambda$ that guarantee the bifurcation of a branch of asymptotically stable $2 \pi$-periodic solutions.

Our theorem 2 complements the previous studies. Indeed, equation (5) is formally different from (2), so the result from [8] cannot be readily applied. The existence and stability of $2 \pi$-periodic solutions of (1) are also discussed in Lazer-McKenna[10] and Fabry[7]. However, it is assumed in [10] that the amplitude of the forcing term $f$ is sufficiently large, while the authors of [7] address those periodic solutions whose amplitude tends to $+\infty$ as a suitable small parameter $\varepsilon>0$ approaches 0 . A degree theoretic approach is developed in [12]. See our survey [11] for a broad analysis of the research around equations of type (5). Extending the range of conclusions about the dynamics of (5) is important as this equation occurs in a variety of applications, e.g. offshore structures [15], resonant screening [16], drilling [6] and others (see [5]).

\section{Lipschitz generalization of the second Bogolubov's theorem}

Throughout the paper $\Omega \subset \mathbb{R}^{k}$ is some open set. For any $\delta>0$ we denote $B_{\delta}\left(v_{0}\right)=\left\{v \in \mathbb{R}^{k}:\left\|v-v_{0}\right\| \leq \delta\right\}$. For any set $M \subset[0, T]$ measurable in the sense of Lebesgue we denote by $\operatorname{mes}(M)$ the Lebesgue measure of $M$. We proved the following result in [5].

Theorem 1 Let $g \in C^{0}\left(\mathbb{R} \times \Omega \times[0,1], \mathbb{R}^{k}\right)$. Let $g_{0}$ be the averaging function given by (4) and consider $v_{0} \in \Omega$ such that $g_{0}\left(v_{0}\right)=0$. Assume that:

(i) For some $L>0$ we have that $\left\|g\left(t, v_{1}, \varepsilon\right)-g\left(t, v_{2}, \varepsilon\right)\right\| \leq L\left\|v_{1}-v_{2}\right\|$ for any $t \in[0, T], v_{1}, v_{2} \in \Omega, \varepsilon \in[0,1]$;

(ii) given any $\gamma>0$ there exist $\delta>0$ and $M \subset[0, T]$ measurable in the sense of Lebesgue with $\operatorname{mes}(M)<\gamma$ such that for every $v \in B_{\delta}\left(v_{0}\right), t \in$ $[0, T] \backslash M$ and $\varepsilon \in[0, \delta]$ we have that $g(t, \cdot, \varepsilon)$ is differentiable at $v$ and $\left\|g_{v}^{\prime}(t, v, \varepsilon)-g_{v}^{\prime}\left(t, v_{0}, 0\right)\right\| \leq \gamma ;$

(iii) $g_{0}$ is continuously differentiable in a neighborhood of $v_{0}$ and the real parts of all the eigenvalues of $\left(g_{0}\right)^{\prime}\left(v_{0}\right)$ are negative.

Then there exists $\delta_{1}>0$ such that for every $\varepsilon \in\left(0, \delta_{1}\right]$, system (3) has exactly one $T$-periodic solution $x_{\varepsilon}$ with $x_{\varepsilon}(0) \in B_{\delta_{1}}\left(v_{0}\right)$. Moreover the solution $x_{\varepsilon}$ is asymptotically stable and $x_{\varepsilon}(0) \rightarrow v_{0}$ as $\varepsilon \rightarrow 0$.

To prove theorem 1 we represent the Poincaré map $P_{\varepsilon}$ of (3) as

$$
P_{\varepsilon}(v)=v+\varepsilon \int_{0}^{T} g(\tau, x(\tau, v, \varepsilon), \varepsilon) d \tau,
$$

where $x(\cdot, v, \varepsilon)$ is the solution $x$ of $(3)$ with the initial condition $x(0)=v$. We then show that condition (iii) ensures that the map

$$
\bar{P}_{\varepsilon}(v)=v+\varepsilon \int_{0}^{T} g(\tau, v, 0) d \tau
$$


contracts in a neighborhood of $v_{0}$, which, in combination with (i) and (ii), allows to conclude that $P_{\varepsilon}$ contracts in a neighborhood of $v_{0}$ too. The later is known to be equivalent to the existence of such a $T$-periodic solution to (3) which originates in the above-mentioned neighborhood of $v_{0}$ and which attracts all other solutions of (3) that originate in this neighborhood. Thus the statement of theorem 1.

\section{Bifurcations of asymptotically stable periodic solutions in differential equations with jumping nonlinearities}

In this section we apply Theorem 1 to studying the bifurcation of asymptotically stable $2 \pi$-periodic solutions in equation (5). A function $u$ is a solution of $(5)$ if and only if $\left(z_{1}, z_{2}\right)=(u, \dot{u})$ is a solution of the system

$$
\begin{aligned}
& \dot{z}_{1}=z_{2}, \\
& \dot{z}_{2}=-z_{1}+\varepsilon\left[-a z_{1}^{+}-c z_{2}+\lambda \cos t\right] .
\end{aligned}
$$

After the change of variables

$$
\left(\begin{array}{l}
z_{1}(t) \\
z_{2}(t)
\end{array}\right)=\left(\begin{array}{cc}
\cos t & \sin t \\
-\sin t & \cos t
\end{array}\right)\left(\begin{array}{l}
x_{1}(t) \\
x_{2}(t)
\end{array}\right)
$$

system (6) takes the form

$$
\begin{aligned}
& \dot{x}_{1}=\varepsilon \sin t\left[a\left(x_{1} \cos t+x_{2} \sin t\right)^{+}+c\left(-x_{1} \sin t+x_{2} \cos t\right)-\lambda \cos t\right], \\
& \dot{x}_{2}=\varepsilon \cos t\left[-a\left(x_{1} \cos t+x_{2} \sin t\right)^{+}+c\left(x_{1} \sin t-x_{2} \cos t\right)+\lambda \cos t\right] .
\end{aligned}
$$

The corresponding averaging function $g_{0}$, calculated according to the formula $(4)$, is

$$
g_{0}\left(x_{1}, x_{2}\right)=\left(\begin{array}{rr}
-\pi c & \pi a / 2 \\
-\pi a / 2 & -\pi c
\end{array}\right)\left(\begin{array}{l}
x_{1} \\
x_{2}
\end{array}\right)+\left(\begin{array}{l}
0 \\
\pi \lambda
\end{array}\right) .
$$

It can be easily checked that the unique zero of $g_{0}$ is

$$
\left(\frac{2 a \lambda}{a^{2}+4 c^{2}}, \frac{4 c \lambda}{a^{2}+4 c^{2}}\right)
$$

and

$$
\text { the eigenvalues of }\left(g_{0}\right)^{\prime} \text { are }-\pi c \pm i \pi a \text {. }
$$

The amplitude of this zero is

$$
A=\frac{2|\lambda|}{\sqrt{a^{2}+4 c^{2}}} .
$$

To apply Theorem 1 it remains to prove the following proposition.

Proposition 1 Let $v_{0} \in \mathbb{R}^{2} \backslash\{0\}$. Then the right hand side of (7) satisfies (ii) for any $c, a, \lambda \in \mathbb{R}$. 
Proof Let $[v]_{i}$ be the $i$-th component of the vector $v \in \mathbb{R}^{2}$. Let $g(t, v)=$ $\left([v]_{1} \cos t+[v]_{2} \sin t\right)^{+}$and notice that it is enough to prove that $g:[0,2 \pi] \times$ $\mathbb{R}^{2} \rightarrow \mathbb{R}$ satisfies (ii). Define $\theta(v)=\arctan \left(-[v]_{1} /[v]_{2}\right)$, if $\left[v_{0}\right]_{2} \neq 0$, and put

$$
\theta(v)=\left\{\begin{array}{lll}
\arctan \left(-[v]_{1} /[v]_{2}\right) & \text { if } & {\left[v_{0}\right]_{1}[v]_{2}<0} \\
\pi / 2 & \text { if } & v=v_{0} \\
\arctan \left(-[v]_{1} /[v]_{2}\right)+\pi & \text { if } & {\left[v_{0}\right]_{1}[v]_{2}>0}
\end{array}\right.
$$

if $\left[v_{0}\right]_{2}=0$. In any case notice that the function $v \mapsto \theta(v)$ is continuous in every sufficiently small neighborhood of $v_{0}$. Fix $\gamma>0$. Let $M$ be the union of the interval $M_{1}$ centered in $\theta\left(v_{0}\right)$ (when $\theta\left(v_{0}\right)<0$, take $\theta\left(v_{0}\right)+2 \pi$ instead of $\left.\theta\left(v_{0}\right)\right)$ and of the interval $M_{2}$ centered in $\theta\left(v_{0}\right)+\pi$, each of length $\gamma / 2$. Take $\delta>0$ such that $\theta(v) \in M_{1}$ for all $v \in B_{\delta}\left(v_{0}\right)$. Of course, also $\theta(v)+\pi \in M_{2}$ for all $v \in B_{\delta}\left(v_{0}\right)$. This implies that for fixed $t \in[0,2 \pi] \backslash M,[v]_{1} \cos t+[v]_{2} \sin t$ has constant sign for all $v \in B_{\delta}\left(v_{0}\right)$. Therefore, $g(t, \cdot)$ is differentiable and $g_{v}^{\prime}(t, v)=g_{v}^{\prime}\left(t, v_{0}\right)$ for all $v \in B_{\delta}\left(v_{0}\right)$. Hence (ii) is fulfilled.

The result of this section can be now summarized as follows.

Theorem 2 Assume that $c>0$ and $A=2|\lambda| / \sqrt{a^{2}+4 c^{2}} \neq 0$ and take arbitrary $R, \delta>0$. Then for each $\varepsilon>0$ sufficiently small, equation (5) has an asymptotically stable $2 \pi$-periodic solution whose amplitude goes to $A$ as $\varepsilon \rightarrow 0$. Moreover, (5) doesn't have $2 \pi$-periodic solutions with amplitudes in

$$
(0, R] \backslash(A-\delta, A+\delta) .
$$

Proof The hypotheses (i) of Theorem 1 is immediate to verify, (ii) is proved in Proposition 1 and (iii) follows from (8). Hence, the existence of a unique branch of asymptotically stable $2 \pi$-periodic solutions whose amplitudes approache $A$ as $\varepsilon \rightarrow 0$ follows from Theorem 1 .

To prove that none of $2 \pi$-periodic solutions of (5) have amplitudes within (10), we recall that the initial conditions of $2 \pi$-periodic solutions of $(5)$ must converge to a zero of the averaging function $g_{0}$ as $\varepsilon \rightarrow 0$, see Buica-LlibreMakarenkov [2, theorem 7 (C13)] (same result under a formal assumption of analiticity was proved in Makarenkov-Ortega [14, lemma 2]). This completes the proof because we earlier noticed that the only zero of $g_{0}$ is that of the amplitute $A$.

Theorem 2 allows deriving the curves of the dependence of the amplitudes of asymptotically stable $2 \pi$-periodic oscillations in (5) upon the parameters, that we have drawn in Fig. 2. In particular, one can see that, for any fixed $\lambda \in \mathbb{R} \backslash\{0\}$, the amplitude tends to $+\infty$ as $\sqrt{a^{2}+4 c^{2}} \rightarrow 0$.

Finally, we note that the case where the period of the perturbation in (5) deviates from $\pi$ slightly (i.e. when we have a detuning, as in the classical Van der Pol oscillator) can be approach over theorem 2 too. Indeed, the change of the variables

$$
v(t)=u((1+\gamma \varepsilon) t)
$$




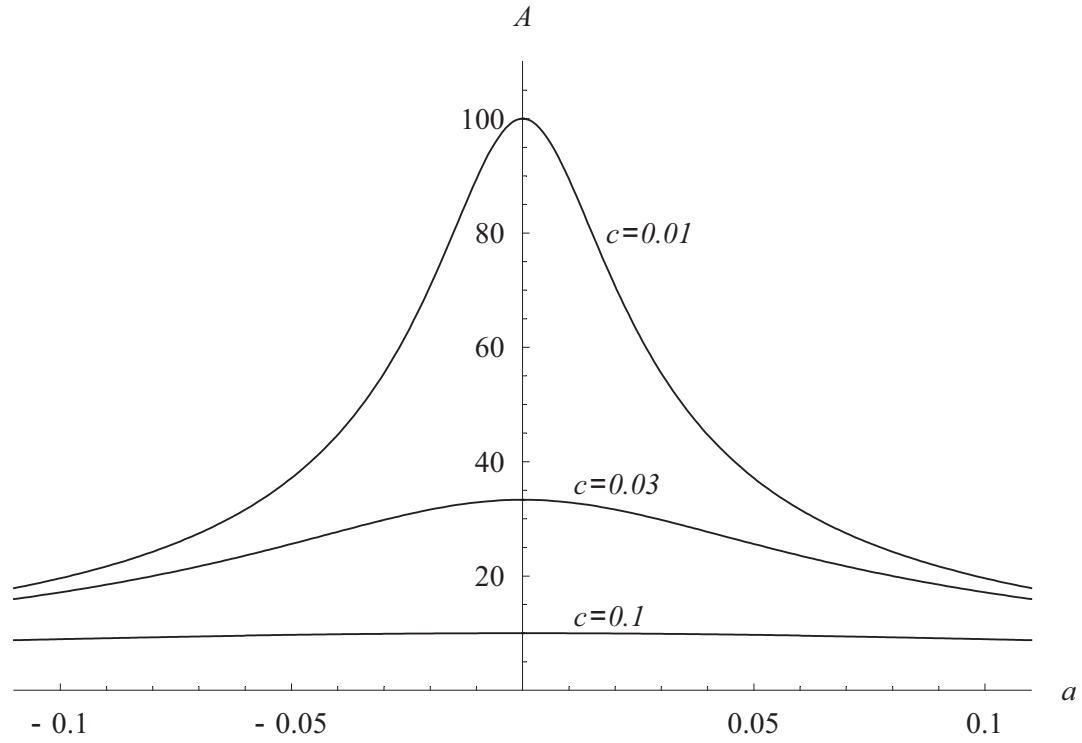

Fig. 2 The curves of dependence of the amplitude of asymptotically stable $2 \pi$-periodic oscillations in (5) upon the parameter $a \in \mathbb{R}$ drawn for fixed $\lambda=1$ and varying values of $c$.

brings the equation with detuning in time

$$
\ddot{u}+\varepsilon c \dot{u}+u+\varepsilon a u^{+}=\varepsilon \lambda \cos \frac{t}{1+\gamma \varepsilon}
$$

to the equation with detuning in the rest of the coefficients

$$
\ddot{v}+\varepsilon c(1+\varepsilon \gamma) \dot{v}+(1+\varepsilon \gamma)^{2} v+\varepsilon a(1+\varepsilon \gamma)^{2} v^{+}=\varepsilon \lambda(1+\varepsilon \gamma)^{2} \cos t .
$$

Literally same arguments as in section 3 apply to investigate asymptotically stable $\pi$-periodic oscillations of equation (11). The only difference is that (11) gives a simple additional term $-\gamma z_{1}$ in the square brackets of system (6), thus formula (9) will contain the parameter $\gamma$ now.

Theorem 1 can be also used for establishing stable resonance oscillations in the case where the unperturbed oscillator is Hamiltonian, e.g. when (5) is of more generic form

$$
\ddot{u}+\varepsilon c \dot{u}+\sin u+\varepsilon a u^{+}=\varepsilon \lambda \cos t
$$

or

$$
\ddot{u}+\sin u=\varepsilon F(t, u, \dot{u}, \varepsilon),
$$

where $F$ is continuous and piecewise smooth in a suitable sense (see Makarenkov [13]). This can be done alone the same lines as the classical Second Bogolyubov's theorem is used for establishing stable resonance oscillations in mechanical oscillators (12) with smooth $F$, see Greenspan-Holmes [9] or Burd $[3]$. 
Acknowledgements The first author is supported by a grant of the Romanian National Authority for Scientific Research, CNCS UEFISCDI, project number PN-II-ID-PCE-20113-0094. The second author is partially supported by a MICINN/FEDER grant number MTM2009-03437, by an AGAUR grant number 2009SGR-410, by an ICREA Academia, two FP7+PEOPLE+2012+IRSES numbers 316338 and 318999. The third author is partially supported by RFBR Grant 13-01-00347. We thank the referees for useful comments which improved our note.

\section{References}

1. Babitsky, V. I. Theory of vibro-impact systems and applications. Translated from the Russian by N. Birkett and revised by the author. Foundations of Engineering Mechanics. Springer-Verlag, Berlin, 1998. xvi+318 pp.

2. A. Buică, J. Llibre, O. Makarenkov, Bifurcations from nondegenerate families of periodic solutions in Lipschitz systems, J. Differential Equations 252 (2012), no. 6, 3899-3919.

3. V. Sh. Burd, Resonance vibrations of impact oscillator with biharmonic excitation. Phys. D 241 (2012), no. 22, 1956-1961.

4. Bogoliubov, N. N.; Mitropolsky, Y. A. Asymptotic methods in the theory of non-linear oscillations. Translated from the second revised Russian edition. International Monographs on Advanced Mathematics and Physics Hindustan Publishing Corp., Delhi, Gordon and Breach Science Publishers, New York 1961, $\mathrm{x}+537$.

5. A. Buică, J. Llibre, O. Makarenkov, Asymptotic stability of periodic solutions for nonsmooth differential equations with application to the nonsmooth van der Pol oscillator. SIAM J. Math. Anal. 40 (2009), no. 6, 2478-2495.

6. Q.-J. Cao, M. Wiercigroch, E. Pavlovskaia, S.-P. Yang, Bifurcations and the penetrating rate analysis of a model for percussive drilling, Acta Mech. Sinica 26 (2010) 467-475.

7. C. Fabry, Large-amplitude oscillations of a nonlinear asymmetric oscillator with damping. Nonlinear Anal. 44 (2001), no. 5, Ser. A: Theory Methods, 613-626.

8. J. Glover, A.C. Lazer, P.J. McKenna, Existence and stability of large scale nonlinear oscillations in suspension bridges. Z. Angew. Math. Phys. 40 (1989), no. 2, 172-200.

9. B. D. Greenspan, P. J. Holmes, Homoclinic orbits, subharmonics and global bifurcations in forced oscillations. Nonlinear dynamics and turbulence, 172-214, Interaction Mech. Math. Ser., Pitman, Boston, MA, 1983.

10. A. C. Lazer, P. J. McKenna, Lazer, A. C.; McKenna, P. J. Existence, uniqueness, and stability of oscillations in differential equations with asymmetric nonlinearities. Trans. Amer. Math. Soc. 315 (1989), no. 2, 721-739.

11. O. Makarenkov, J. S. W. Lamb, Dynamics and bifurcations of nonsmooth systems: a survey. Phys. D 241 (2012), no. 22, 1826-1844.

12. O. Makarenkov, P. Nistri, Periodic solutions for planar autonomous systems with nonsmooth periodic perturbations. J. Math. Anal. Appl. 338 (2008), no. 2, 1401-1417.

13. O. Makarenkov, The asymptotic stability of the oscillations of a two-mass resonance sifter, Journal of Applied Mathematics and Mechanics 77 (2013), no. 3, 287 - 295.

14. O. Makarenkov, R. Ortega, Asymptotic stability of forced oscillations emanating from a limit cycle. J. Differential Equations, 250 (2011), no. 1, 39-52.

15. J. M. T. Thompson, H. B. Stewart, Nonlinear dynamics and chaos. Second edition. John Wiley \& Sons, Ltd., Chichester, 2002. xxii+437 pp.

16. B. Wen, Recent development of vibration utilization engineering, Front. Mech. Eng. Chin. 3 (1) (2008) 1-9. 